\title{
Selección de los recursos humanos de la actividad física y del deporte en las empresas
}

\section{Selection of human resources of physical activity and sport in companies}

\author{
Antonio Campos Izquierdo ', María Dolores González Rivera ${ }^{2}$ \\ ' Facultad de Ciencias de la Actividad Física y del Deporte-INEF. Universidad Politécnica de Madrid. \\ ${ }^{2}$ Departamento de Psicopedagogía y Educación Física. Universidad de Alcalá \\ antonio.campos.izquierdo@upm.es marilin.gonzalez@uah.es
}

Fecha de recepción: 17-11-2009

Fecha de aceptación: 18-06-2010

Resumen: Este artículo analiza algunos elementos del proceso de selección de los recursos humanos de la actividad física y del deporte en las empresas de la Comunidad Valenciana. En este estudio se obtiene que el reclutamiento lo realizan las propias empresas y es esencialmente externo. Asimismo, en la selección esencialmente se utilizan como instrumentos el currículum vitae, las referencias o contactos y la entrevista. Además, la mayoría de los seleccionados no tienen la correspondiente titulación, lo que determina que, junto al elevado número de seleccionados por referencias o contactos, dicho proceso en muchos casos es inadecuado y con escasa fiabilidad y validez.

Palabras clave: selección, recursos humanos, empresas, deporte, reclutamiento.

Abstract: This research analyzes the process of selection of the human resources of the physical activity and sport in the companies of the Region of Valencia. In this research is obtained that the own companies realize the recruitment and is essentially external. Likewise, in the selection essentially they use as instruments the curriculum vitae, the references or contacts and the interview. Besides, the majority of the selected ones do not have the corresponding qualifications and there is a high number of selected by references or contacts, determines that the above mentioned process in many cases is inadequate and with scanty reliability and validity.

Key Words: selection, human resources, companies, sport, recruiting.

\section{Introducción}

La sociedad está constituida, integrada y estructurada por una inmensidad y diversidad de organizaciones. Todas estas organizaciones tienen un elemento común y fundamental que son las personas, y sin ellas, las propias organizaciones no existirían, siendo además las propias personas las que caracterizan y conforman cada organización.

Además, «en el contexto de alta competencia en el que se desenvuelve la actividad económica actual, las personas y la forma en que éstas son dirigidas adquieren cada vez más importancia» (De Saá y García, 200 l : I I I), donde los recursos humanos pueden desempeñar una fuente esencial de una ventaja competitiva sostenible y estratégica así como ser un factor clave de creación de valor en las empresas (Becker, Huselid y Ulrico, 2001) y no ser considerados simplemente como un coste (Pfeffer, 1994).
Al hacer referencia al término recursos humanos se está hablando de las personas, desde toda su amplitud y concepción, que trabajan y desarrollan su actividad laboral en los distintos tipos de organizaciones. Si se profundiza en este término, Fernández-Ríos ( 1999 ) explica que los recursos humanos son los conocimientos, capacidades, experiencias, habilidades y compromisos de los empleados de las distintas organizaciones.

Por su parte, los recursos humanos han estado presentes, de una forma u otra, en todos los enfoques y teorías organizativas explicadas, entre otros, por Renau (1985), Cohen (1991), Chiavenato (2006) y Kootnz y Weihrich (2007). Pero el concepto de dirección y organización de los recursos humanos actual no nace hasta mediados del siglo pasado, el cual ha ido evolucionando (Gasalla 1993; Claver et al. 1996) hasta la concepción actual en la que se considera que los recursos humanos deben estar integrados en to- 
das las estrategias, procesos y actuaciones de la organización teniendo un papel central (Wright y Snell, 1991; Schuler, 1992; Pereda y Berrocal, 1999; Becker, Huselid y Ulrich, 200 I; Porret, 2006) y como elemento fundamental de la excelencia de las empresas (Fundación Europea para la gestión de la Calidad, 2003). Esta evolución ha supuesto el paso de un enfoque micro, que ha dominado la investigación sobre recursos humanos en el pasado, al enfoque actual más macro o estratégico (Delery y Doty, 1996).

Todo ello ha generado en España, en las últimas décadas, numerosas investigaciones que han tenido como tema central los recursos humanos en las empresas (Pérez y Garrido, 2006). Sin embargo, en el ámbito de la actividad física y del deporte las investigaciones sobre recursos humanos todavía son muy escasas (Martínez del Castillo, 1997; Campos, 2007).

Al respecto, los recursos humanos de las organizaciones de la actividad física y del deporte se encuentran principalmente en el sector servicios, el cual, según el Instituto Nacional de Estadística en el año 2008, representaba el 68, I\% de las personas ocupadas. Pero específicamente los recursos humanos en la actividad física y el deporte, aunque es difícil de cuantificar y de delimitar (Heinemann, 1998 y Camy et al. 1999), en Europa representan alrededor del 2\% de los puestos de trabajo (Andreff y Weber, 1995). Por su parte, en España se estima en 200.000 los empleos directos sobre actividad física y deporte en España, a lo que habría que sumarle los empleos indirectos (Consejo Superior de Deportes, 2000).

Asimismo, existe una gran diversidad de organizaciones que ofrecen y desarrollan servicios de actividad física y deporte en los que trabajan los recursos humanos, como son las diferentes administraciones públicas, las distintas entidades privadas asociativas y las empresas, las cuales, estas últimas, son el objeto de esta investigación. Dentro de estas organizaciones, Campos $(2007,2010)$ clasifica y estructura a los recursos humanos en dos grupos:

- Los recursos humanos indirectos de la actividad física y del deporte, que trabajan en servicios de actividad física y deporte pero que realizan funciones laborales no especificas de la actividad física y del deporte (personal sanitario, de mantenimiento, etc.).

- Los recursos humanos de la actividad física y del deporte, que son el objeto de esta investigación, que trabajan desarrollando funciones laborales de la actividad física y del deporte, que deben poseer la correspondiente titulación de la actividad física y del deporte y que pertenecen a las profesiones, establecidas en el anteproyecto de Ley de Ordenación del Ejercicio de las Profesiones del Deporte (Consejo Superior de Deportes, 2009), como son: Monitor Deportivo, Entrenador Profesional, Profesor de Educación Física y Director Deportivo.

Existen diversas investigaciones sobre los recursos humanos de la actividad física y del deporte que estudian, al nivel general, los aspectos demográficos, laborales, estructurales y formativos, tales como Martínez del Castillo ( I99 I), Camy et al. (1999), Campos (200 I), Madella, (2002), Augustin (2003), Tojal (2004). Otras investigaciones se han centrado en profesiones concretas como es el caso de los directores deportivos (Quain y Parks, 1986; García Ferrando, 1986; López y Luna-Arocas, 2000; Gómez y Mestre, 2005), los monitores deportivos (Comisiones Obreras, 2000; González, 2008; Moreno y Gutiérrez, I999), los entrenadores (Seirul-lo, 1987; Shibli et al., 1999) o profesores de Educación Física (Puig, 1980; McMurtie; 1997; Hardman y Marshall, 2000). Sin embargo, son muy escasos los estudios que se centran únicamente en la propia dirección y organización de los recursos humanos o en algunos de los distintos elementos de su proceso, como pueden ser: el análisis del puesto de trabajo del director (Peiró et al., 1999), los estilos y modelos de gestión (Ramos, 1993), la estructura organizativa (Gambau, 2007) o el proceso de selección. Este último es el objeto de este estudio, que aunque se pretende dar una visión integral, se centra en un aspecto esencial y concreto que ha sido escasamente estudiado en la dirección y organización de los recursos humanos de la actividad física y del deporte en las empresas.

\section{El proceso de selección de los recursos humanos de la actividad física y del deporte}

En la dirección y organización de los recursos humanos de la actividad física y del deporte, uno de los elementos más importantes es la selección de personas, la cual está constituida por los procesos de reclutamiento, selección e incorporación de las personas.

El proceso de selección parte de la necesidad inmediata, a corto, medio o largo plazo de incorporar a personas en uno o varios puestos de trabajo, generando el proceso de reclutamiento, el cual es esencial para la selección. Esto es debido a que se pre- 
tende obtener un número suficiente de candidatos, en el momento oportuno, que posean los requisitos mínimos para el puesto de trabajo que se pretende cubrir y entre los cuales poder elegir (Peiró, 1992; Claver et al., 1996; Bayón, 2002; Dolan et al., 2003 y Sastre y Aguilar, 2003). Aunque, en las organizaciones de la actividad física y del deporte, no se le suele dar importancia a este proceso ni asignar medios suficientes, tanto económicos, materiales como personales (Soucie, 2002; Pires, 2007). Asimismo, Peiró (1992) expone que en el proceso de reclutamiento no sólo es importante la cantidad de personas atraídas, sino también el nivel de cualificación en función del puesto de trabajo. Además, una de las etapas más importantes del reclutamiento es la identificación, selección y mantenimiento de las fuentes que pueden ser proveedoras de candidatos (Dolan et al., 2003).

En el proceso de reclutamiento se puede diferenciar el interno (cuando el proceso está orientado hacia personas pertenecientes a la propia organización) o el externo (cuando el proceso está orientado hacia personas externas de la organización), siendo el reclutamiento externo mucho más utilizado que el interno en las organizaciones de la actividad física y del deporte (Martínez del Castillo, 199I; MacLean, 200 I; Lussier y Kimball, 2008). En España, al nivel general, los métodos de reclutamiento externo más utilizados son: el reclutamiento informal a través de empleados o conocidos, mediante anuncios, el recurrir a agencias de trabajo y a través de Internet (Salgado, Moscoso y Lado, 2004). Respecto al uso de Internet, el reclutamiento externo, en la última década ha sufrido un cambio profundo con el aumento de utilización de Internet, tanto desde la propia página corporativa como en la difusión o búsqueda de candidatos en portales o páginas web de empleo,ya que se consigue reducir considerablemente los costes temporales y económicos y se proporciona más información acerca de la company and specific career opportunities empresa y las oportunidades de las personas (Pin, Laorden y Sáenz-Díez, 2002; Paz, 2004; Lussier y Kimball, 2008).

Posteriormente, With such information in hand, they can then los candidatos elegidos en el reclutamiento, comienzan el proceso de selección específico a través del cual se elige el más adecuado para el puesto de trabajo en el que existe la necesidad de incorporación (Peiró, 1992; Pereda y Berrocal, 1999). En las organizaciones de actividad física y deporte, principalmente dicho proceso es realizado por la propia organización, en vez de subcontratar a una empresa especialista en selección (Martínez et al., 2008; Campos, 2010).
En la selección es preciso emplear instrumentos evaluativos, porque sino será un proceso de incorporación en vez de selección (Salgado, Moscoso y Lado, 2004). Existe una gran variedad de instrumentos para llevar a cabo la selección como son, entre otros, la entrevista personal, currículum vitae, las pruebas profesionales, las pruebas psicotécnicas, las pruebas situacionales, la entrevista de incidentes críticos, las dinámicas de grupo y los assessment centers (Levy-leboyer, 1992; Pereda y Berrocal, 200 I). En España, los instrumentos más utilizados en la selección, a nivel general, son: entrevista, currículum vitae, test de habilidades cognitivas y psicomotoras, test y cuestionarios de personalidad, referencias, test de muestra de trabajo y assessment centers (Salgado, Moscoso y Lado, 2004). Por su parte, en América Latina el reclutamiento y selección de personas se basa generalmente en rasgos de la personalidad y características físicas de la persona (Elvira y Davila, 2005).

En el caso de las organizaciones de actividad física y deporte los instrumentos utilizados en la selección son escasos y tradicionales, siendo los mayoritarios el currículum vitae y los contactos (Martínez del Castillo, 1991; Heinemann, 1998; Augustin, 2003; Puig y Viñas, 2006). Además, existe un elevado porcentaje de personas seleccionadas que no tienen alguno de los diferentes tipos de titulaciones de la actividad física y del deporte (Camy, el al., 1999; Campos, 2007; González, 2008). Al respecto, hay que recodar que la actividad física y el deporte genera beneficios en las personas respecto a su salud, educación, aspectos éticos y sociales, pero si ésta no es dirigida, diseñada, desarrollada o evaluada por una persona con la titulación de la actividad física y del deporte específica y competente, estos beneficios se pueden convertir en perjuicios y riesgos para la salud, la seguridad y la educación de los ciudadanos y para la sociedad; además de determinar la calidad y eficiencia de los propios servicios.

También, hay que enfatizar que es necesario mantener un constante $y$ actualizado seguimiento $y$ evaluación de cada uno de los procedimientos, técnicas e instrumentos que se utilizan en el proceso de selección, para examinar su validez y fiabilidad, así como su utilidad a la hora de predecir el futuro desempeño de los candidatos (Pereda y Berrocal, 1999; Salgado, Moscoso y Lado, 2004). En este sentido, Luna (2000), en función de la validación del proceso de selección, habla de validez predictiva, concurrente y de contenido y constructo.

Una vez seleccionada la persona idónea, se procede a su incorporación al puesto de trabajo, en la que, 
durante un tiempo determinado, se encuentra en periodo de prueba, el cual es una parte fundamental del proceso de selección ya que se comprueba, mediante una constante y adecuada evaluación del desempeño en situación real si es la persona adecuada para el puesto de trabajo (Pereda y Berrocal, 1999; Campos, 2010). Si la persona supera el periodo de prueba, seguirá en la organización, por el contrario sino lo supera, volverá a iniciar el proceso de selección, al igual que cuando se produzca la sustitución por baja o la desvinculación de la organización.

También, hay que señalar que la selección y el reclutamiento parten del análisis y descripción del puesto de trabajo respectivo, en el que el apartado de requisitos, exigencias y necesidades establecido tiene una importancia crucial, y donde la formación inicial, entre otras consideraciones, es un aspecto fundamental (Bayón, 2002; Chiavenato, 2007; Campos, 2010), así como las competencias determinadas (McClelland, 1973; Boyatzis, 1982).

Por todo ello, y considerando la importancia y relevancia del proceso de selección en la dirección y organización de los recursos humanos de la actividad física y del deporte, esta investigación analiza el proceso de selección de los recursos humanos de la actividad física y del deporte en las empresas de la Comunidad Valenciana en los años 2004 y 2006. Los objetivos específicos de esta investigación con respecto a los recursos humanos de actividad física y deporte que trabajan en empresas que ofertan y desarrollan servicios de actividad física y deporte en la Comunidad Valenciana son:

- Determinar la tipología del reclutamiento en los años 2004 y 2006.

- Describir los instrumentos utilizados en la selección en los años 2004 y 2006.

- Establecer la formación inicial de los recursos humanos seleccionados en los años 2004 y 2006.

- Describir los instrumentos utilizados en la selección según la formación inicial de los recursos humanos en los años 2004 y 2006.

\section{Metodología}

La metodología que se ha seguido en esta investigación ha sido cuantitativa de corte descriptivo (Alvira, 2004). En el desarrollo de esta metodología, los procedimientos que se han seguido son los propios de una encuesta seccional (Cea, 1998; Corbetta, 2003), aplicada a una muestra de personas que trabajan desarrollando funciones laborales de la actividad física y del deporte en la Comunidad Valenciana (tabla I). La encuesta se ha realizado mediante la realización de entrevista personal estandarizada por medio de cuestionario en las instalaciones deportivas a cada una de las personas seleccionadas de una muestra estadísticamente representativa.

Para establecer el universo objeto de estudio, el número de personas que trabajaban en funciones de la actividad física y del deporte (tabla I), se ha seguido el estudio de Martínez del Castillo (1992) sobre la proyección del mercado laboral de la actividad física y del deporte, ya que, como explica Heinemann ( 1999) y Madella (2003), los puestos de trabajo referidos a este mercado de trabajo no se recogen estadísticamente de forma específica y por ello es difícil conocer exactamente su cuantificación, sino que han de deducirse de diversos cálculos, encuestas y estadísticas. El universo que se obtuvo fue de 10.000 personas que trabajaban en funciones de la actividad física y del deporte en la Comunidad Valenciana (tabla I).

Las personas que desarrollan funciones de la actividad física y del deporte trabajan para diferentes tipos de organizaciones (administraciones públicas, entidades privadas asociativas y empresas). En el caso de esta investigación, las entidades objeto de estudio son la empresas que ofertan y desarrollan servicios de la actividad física y del deporte, que en el año 2004 eran 1.018 empresas, de las que en la provincia de Valencia eran 540 (53\% de la empresas), en la provincia de Alicante 339 (33,3\% de la empresas) y en la provincia de Castellón 139 ( 13,7\% de las empresas), y en el año 2006 eran I. 109 empresas, de las que en la provincia de Valencia eran 592 (53,4\%), en la provincia de Alicante $376(33,9 \%)$ y en la provincia de Castellón I4| (12,7\%).

\section{I. Muestra}

El tamaño de la muestra en cada uno de los años estudiados fue de 600 personas que trabajaban en funciones de la actividad física y del deporte en la Comunidad Valenciana. Dado una población de 10.000 personas según el estudio de Martínez el Castillo ( 1992), y trabajando con un intervalo de confianza del $95,5 \%$, y suponiendo en la varianza poblacional el caso más desfavorable de p igual a 50\%, luego q= $50 \%$, el margen de error permitido de muestreo es de $\pm 4 \%$. 
Tabla 1

Funciones laborales de la actividad física y del deporte delimitadas en este estudio

\begin{tabular}{|l|}
\hline \multicolumn{1}{|c|}{ FUNCIONES DE LA ACTIVIDAD FÍSICA Y DEL DEPORTE } \\
\hline Impartir docencia de actividad física y deportes. \\
Impartir docencia de Educación Física y deportes dentro de las enseñanzas de régimen general, régimen especial y universitaria. \\
Entrenar equipos e individuos para la competición y para la superación de pruebas físicas. \\
Desarrollar entrenamiento personalizado. \\
Impartir docencia de actividades físico-deportivas extraescolares en centros escolares. \\
Desarrollar animación y recreación deportiva. \\
Cuidar y mantener la forma física en grupos. \\
Inspeccionar sobre actividad física y deporte. \\
Dirigir, organizar y coordinar actividad física y deporte. \\
Asesorar, investigar y certificar sobre actividad física y deporte. \\
Readaptar y reeducar físicamente mediante ejercicio físico. \\
Vigilar y socorrer en el medio acuático (socorrismo acuático).
\end{tabular}

La afijación de la muestra, fue proporcional a la distribución de las personas según tamaño demográfico de los municipios y situación geográfica. Además, se realizaron diversos submuestreos teniendo como objetivo dispersar la muestra para que influyera positivamente en la precisión de las estimaciones (Sierra, 200 I; Rodríguez, 1991). El tipo de muestreo fue probabilístico de tipo polietápico, estratificado en primera fase, por conglomerados (Fink, 1995; Miquel et al., 2000; Bryman, 2004), siendo las unidades de primera etapa las provincias en que residían habitualmente las personas. El resto de unidades de etapa han sido en el siguiente orden hasta llegar a las unidades últimas, es decir las personas que trabajan en funciones de la actividad física y del deporte a entrevistar. Estas etapas son las siguientes (dentro de cada provincia seleccionada): comarca, municipio, instalación deportiva, en la que se localizó, entrevistó e identificó a la persona objeto de estudio.

Los 600 sujetos participantes en el estudio, en cada año, que trabajaban para los diferentes tipos de organizaciones de la actividad física y del deporte (administraciones públicas, entidades privadas asociativas y empresas), en el año 2004, el 5 I\% trabajaban para empresas, y en el 2006 son el 52,7\%. Asimismo, dentro de la representatividad de la muestra de las personas que trabajan en funciones de la actividad física y del deporte en las empresas atendiendo a la naturaleza público/privada de las instalaciones deportivas, en el año 2004 son el 51,2\% las personas que trabajan para empresas en una instalación pública y en el año 2006 son el 54,6\% las que trabajan en una instalación pública.

\subsection{Instrumento}

Siguiendo a Miquel et al. (2000) y García Ferrando (2002), una vez establecidos los objetivos, se elaboró y validó el instrumento utilizado para obtener la información, que fue una entrevista de tipo oral, individual y estandarizada por medio de cuestionario compuesta de preguntas cerradas y categorizadas (Fink, 1995; Bryman, 2004).

Para captar la información necesaria para los objetivos del estudio, se decidió partir del «Cuestionario de situación profesional de los recursos humanos de la actividad física y del deporte» (Campos, 2003) para medir el proceso de selección de dichas personas y otras variables relacionadas y ya validado en estudios previos sobre recursos humanos de la actividad física y del deporte (Campos, 2000; Campos, 200 I). En relación a las características psicométricas de este instrumento, el grado de validez ha quedado establecido a través de las opiniones de los expertos, asentado en la bibliografía y otros criterios que provienen del propio instrumento (como son las entrevistas estructuradas realizadas) y la fiabilidad probada tras la comprobación de la escasa variación de las observaciones obtenidas en las diversas aplicaciones del instrumento. En este sentido, en cuanto a la validez de contenido, se llevó a cabo una serie de fases, siguiendo a autores como Cea (200I) y Alvira (2004), que determinan que este instrumento es fiable y válido: en primer lugar, en la fase de diseño del cuestionario y debido a que no se encontró un cuestionario específico durante la fase de revisión bibliográfica que abarcara todos los objetivos del estudio, 
se utilizaron diversos cuestionarios, de los cuales se escogieron las variables que más se adecuaban a los objetivos de la investigación, se tomaron algunas preguntas sin modificar, otras se adaptaron, y el resto de las cuestiones se elaboraron durante el proceso del diseño del cuestionario. Entre los diversos cuestionarios, se tomaron como principal referencia (por considerar que están más en concordancia con los objetivos del estudio) los elaborados por Martínez del Castillo ( I99|), Rivadeneyra (2000), Puig y Viñas (200l) y Campos (200I). Además, se llevó a cabo los grupos de discusión, siguiendo las pautas determinadas por Miquel et al. (2000), ya que esta técnica es útil durante la fase de exploración de cualquier investigación, y ayuda en la fase del diseño del instrumento de obtención de datos, debido a que las discusiones de grupo suministran informaciones valiosas para la elaboración y validación de cuestionarios eficaces (Madella, 2003). Seguidamente, se determinaron las dimensiones y variables para la organización del contenido del instrumento de obtención de datos y, a continuación, se realizó un proceso de selección, elaboración y organización de las preguntas del cuestionario, con sus posibles opciones de respuestas. Esta primera fase de diseño del instrumento dio como resultado la entrevista estandarizada por medio de cuestionario inicial que, tras la realización de su validación a través del juicio de expertos y del estudio piloto y sus posteriores mejoras según lo obtenido, se estableció la entrevista estandarizada por medio de cuestionario definitiva. En cuanto al juicio de expertos, fue valorado por diez especialistas externos al equipo de investigación los cuales evaluaron positivamente el instrumento. En el pretest, el instrumento fue administrado a cincuenta personas pertenecientes al universo estudiado, esta cantidad se encuentra dentro de los márgenes (entre 30 y 50 entrevistas) detallados por Cea (2001) o García Ferrando (2002). Igualmente, sobrepasa el 10\% de la muestra estipulado por Sierra (200I) y Alvira (2004). Todos los sujetos entendieron las cuestiones y alternativas de respuesta, y no hubo valores perdidos.

Además, este instrumento de recogida de datos posee una fiabilidad de consistencia, lo que le da una estabilidad, ya que se ha utilizado posteriormente en diferentes investigaciones como las de Martínez et al. (2008), González (2008) y Espada, Campos y González (2009).

\subsection{Procedimientos}

La investigación ha sido de corte transversal (Miquel et al., 2000), debido a que la recogida de la informa- ción se llevó a cabo durante los dos segundos semestres de los años 2004 y 2006. Toda la fase de procedimiento de recogida de la información (localización, identificación y contacto con las personas que iban a ser entrevistadas, la realización de la entrevista, registro de información y la recogida de la información obtenida), en cada uno de los años, fue realizada por un único entrevistador, con la finalidad de obtener una mayor fiabilidad y validez en el estudio (García Ferrando, 2002). Las 600 entrevistas, en cada año, se realizaron de forma personal e individual cara a cara a cada uno de los individuos seleccionados de la muestra en las instalaciones (Fink, 1995; Bryman, 2004) y tuvieron una duración promedio de quince minutos.

Los análisis de datos han sido efectuados, tras ser tabulados y mecanizados informáticamente. Se ha realizado un análisis descriptivo univariable y bivariable, así como un análisis inferencial a través de tablas de contingencia que incluyen el valor de Chi-cuadrado de Pearson y su significación, así como el coeficiente de correlación Phi. Todo ello empleando el paquete de programas SPSS para WINDOWS (V |4.0).

\section{Resultados y discusión}

En este estudio se obtiene que dentro de los diversos tipos de organizaciones que ofertan y desarrollan servicios de la actividad física y del deporte (administraciones públicas, entidades privadas asociativas y empresas) en la Comunidad Valenciana en el año 2004 , el $51 \%$ de los recursos humanos de la actividad física y del deporte trabajaban para empresas y en el 2006 este porcentaje es levemente superior (52,7\%). Estos porcentajes son superiores al 35,2\% obtenido por Martínez del Castillo ( 199 |), lo que corrobora el aumento en las últimas décadas de las empresas, debido principalmente a la mayor diversificación e incremento de los servicios de la actividad física y del deporte y de su profesionalización, así como al aumento de la subcontratación de empresas por parte de los ayuntamientos en los servicios de la actividad física y del deporte.

Además, en el año 2004 el 17,7\% de los recursos humanos de la actividad física y del deporte eran empleados por cuenta propia y en el 2006 este porcentaje es superior (23,2\%). Ambos porcentajes son superiores al I I,5\% obtenido en el estudio de Martínez del Castillo ( 199 |), lo que corrobora la opinión de Camy et al. (1999) y Puig y Viñas (2006) sobre el aumento del autoempleo en la actividad física y el deporte en las últimas décadas en España. Al respec- 
to, hay que tener en cuenta que «el empleo por cuenta propia se considera una fuente importante de nuevos puestos de trabajo, así como una alternativa al empleo asalariado» (Capelleras y Genescá, 2004:73).

\section{I. El reclutamiento de los recursos humanos de la actividad física y del deporte}

Respecto al reclutamiento, hay que señalar que en todos los casos estudiados éste es realizado por las propias empresas, por lo que dichas empresas no subcontratan a otras entidades para realizar dicho proceso, al igual que obtienen Campos (2003) y González (2008). La tipología del reclutamiento, principalmente es externa (93,5\% en 2004 y 94,3\% en 2006), como también determinan Martínez del Castillo (1991), MacLean (2001) y Lussier y Kimball (2008) (tabla 2).

Tabla 2

Tipología del reclutamiento

\begin{tabular}{|l|c|c|}
\hline & $\mathbf{2 0 0 4}(\mathbf{\% )}$ & $\mathbf{2 0 0 6}(\%)$ \\
\hline Reclutamiento externo & 93,5 & 94,3 \\
\hline Reclutamiento interno & 6,5 & 5,7 \\
\hline
\end{tabular}

En cuanto al reclutamiento externo, en la inmensa mayoría de las empresas el proceso es realizado en su totalidad por la propia entidad (98, I\% en 2004 y $97,7 \%$ en 2006) y principalmente ha sido mediante la difusión de la información en instalaciones deportivas o en centros de formación, a través de sus empleados o conocidos, y mediante la utilización de lnternet (en su página web corporativa o en páginas web de empleo de Internet). Este último método de reclutamiento está en expansión debido a su menor coste económico y temporal (Pin, Laorden y SáenzDíez, 2002; Paz, 2004; Salgado, Moscoso y Lado, 2004; Lussier y Kimball, 2008). El hecho de recurrir a otras entidades en una parte del proceso, al utilizar las bolsas de trabajo de las oficinas de empleo y del Colegio Profesional, es escaso debido a que sólamente representa alrededor del 2\% en ambos años (Tabla 3).

\subsection{La selección de los recursos humanos de la actividad física y del deporte}

El instrumento en la selección de los recursos humanos de la actividad física y del deporte más utilizado
Tabla 3

Reclutamiento externo

\begin{tabular}{|l|c|c|}
\hline & $\mathbf{2 0 0 4}$ (\%) & $\mathbf{2 0 0 6}$ (\%) \\
\hline $\begin{array}{l}\text { Realizado por la propia empresa en } \\
\text { su totalidad }\end{array}$ & 98,1 & 97,7 \\
\hline $\begin{array}{l}\text { Realizado una parte del proceso por } \\
\text { otra entidad }\end{array}$ & 1,9 & 2,3 \\
\hline
\end{tabular}

es el currículum vitae (38,5\% en 2004 y $41,4 \%$ en 2006), seguido de las referencias o contactos (31,7\% y $38,1 \%$, respectivamente), y de las entrevistas (29,2\% y $20,5 \%$, respectivamente) (tabla 4 ). Todo ello confirma, siguiendo a Salgado y Moscoso (2008), que las empresas realizan esencialmente un proceso de selección tradicional, al utilizar únicamente el currículum vitae, las referencias o contactos y las entrevistas. Esto indica que no se utiliza la variedad de herramientas en los diferentes tipos de empresas en España expuesta por Salgado et al. (2004), Levy-Levoyer (1992) y Pereda y Berrocal (200 I). Igualmente, no se utiliza ningún instrumento orientado a la selección por competencias instaurada por McClelland (1973).

El elemento denominado «referencias o contactos» es muy diversificado, ya que va desde las referencias positivas de un empleado sobre una persona, pasando por haber sido alumno o cliente de la entidad, hasta lo que denominan muchas de las personas entrevistadas «enchufe». Al respecto, autores como Martínez del Castillo ( 199 I), Camy et al. ( 1999), Garrigós, (2002), Tojal (2004) y González (2008) expresan que éste es un elemento generalizado para la selección en la actividad física y el deporte. Esto ocasiona, en muchos casos, incertidumbre sobre la adecuación y lógica de dicho proceso y de los criterios utilizados y más aún si se realiza sin tener en cuenta la formación inicial correspondiente (titulación de la actividad física y del deporte), tal y como se describe posteriormente.

Tabla 4

Instrumentos utilizados en la selección

\begin{tabular}{|l|c|c|}
\hline & $\mathbf{2 0 0 4}(\%)$ & $\mathbf{2 0 0 6}(\%)$ \\
\hline Currículum vitae & 38,5 & 41,4 \\
\hline Referencias o contactos & 31,7 & 38,1 \\
\hline Entrevista & 29,2 & 20,5 \\
\hline Pruebas físicas & 0,6 & 0 \\
\hline
\end{tabular}


Un aspecto que se debe destacar, es que en la selección principalmente se utiliza solamente un instrumento (71,2\% de las personas en 2004 y 69,7\% en 2006), y en este caso, los más utilizados son las referencias o contactos (45,8\% de las personas en 2004 y $54 \%$ en 2006 ) y el currículum vitae con el $35,1 \%$ (35, 1\% de las personas en 2004 y 39,7\% en 2006). Después, con menores porcentajes, está la utilización conjunta de dos instrumentos (25,5\% de las personas en 2004 y 27,7\% en 2006), siendo el mayoritario cuando se utiliza conjuntamente el currículum vitae y la entrevista (73,3\% y 64,3\% de las personas, respectivamente). Sólamente se utilizaron tres instrumentos en el 3,3\% de las personas en 2004 y en el 2,6\% en 2006, mayoritariamente, la utilización conjunta de currículum vitae, entrevista y referencias o contactos (75,8\% en el año 2004 y l00\% en el año 2006) (Tabla 5).

Tabla 5

Cantidad de instrumentos utilizados en la selección

\begin{tabular}{|l|c|c|}
\hline & $\mathbf{2 0 0 4}(\%)$ & $\mathbf{2 0 0 6}(\%)$ \\
\hline Utilización de un instrumento & 71,2 & 69,7 \\
\hline Utilización de dos instrumentos & 25,5 & 27,7 \\
\hline Utilización de tres instrumentos & 3,3 & 2,6 \\
\hline
\end{tabular}

\subsection{La formación inicial de los recursos humanos de la actividad física y del deporte}

El reclutamiento y la selección parten del análisis y descripción del puesto de trabajo respectivo, donde el apartado de requisitos y exigencias tiene una importancia crucial (Fernández-Ríos, 1995; Bayón, 2002). Dentro de éste, la formación inicial, entre otras consideraciones, es un aspecto fundamental, y en el caso de los recursos humanos de la actividad física y del deporte, debe ser la titulación de la actividad física y del deporte correspondiente. Por ello, en el proceso de selección debe ser un criterio primordial y básico (Martínez del Castillo, 1991; Camy et al., 1999, Tojal, 2004; Consejo Superior de Deportes, 2009). Asimismo, se debe enfatizar que este criterio, aunque es esencial, no es el único, sino que posteriormente se deben valorar otros aspectos (Campos, 2010).

Si se analiza la formación inicial de los recursos humanos de la actividad física y del deporte se obtiene que el 49,8\% en el año 2004 y el 48,7\% en 2006 son personas que no poseen ninguna de las diferen- tes titulaciones de la actividad física y del deporte (Tabla 6). Además, hay que resaltar que estos porcentajes son muy superiores al 36,7\% obtenido por Martínez del Castillo ( 99 |), lo que muestra que, en el proceso de selección, el número de personas elegidas sin formación inicial ha aumentado en las últimas décadas.

Tabla 6

Formación inicial de las personas seleccionadas

\begin{tabular}{|l|c|c|}
\hline & 2004 (\%) & 2006 (\%) \\
\hline $\begin{array}{l}\text { Titulación de la actividad física } \\
\text { y del deporte }\end{array}$ & 50,2 & 51,3 \\
\hline $\begin{array}{l}\text { No titulación de la actividad física } \\
\text { y del deporte }\end{array}$ & 49,8 & 48,7 \\
\hline
\end{tabular}

Además, este porcentaje de personas no seleccionadas adecuadamente es mayor, ya que la mayoría de las personas seleccionadas tituladas de la actividad física y del deporte (55,7\% en 2004 y 60,5\% en 2006) realizan funciones para las que su formación en la titulación que poseen no les capacita adecuadamente (por ejemplo: un técnico deportivo de fútbol que enseña natación). Esto puede ser debido a que muchas personas que seleccionan consideran la incongruencia de que todas las titulaciones de la actividad física y del deporte sirven para todas las funciones. Todo ello, determina que sólamente alrededor del $20 \%$ de los recursos humanos de la actividad física y del deporte tienen titulación de la actividad física y del deporte y realizan funciones para las que son competentes.

Todo lo anterior establece que el proceso de selección de los recursos humanos en las empresas de la actividad física y del deporte en muchos casos es inadecuado y con escasa fiabilidad y validez; y más cuando una gran parte de las personas seleccionas es por referencias o contactos, ya que se da a entender que la actividad física y el deporte puede ser desarrollada por cualquier persona, por lo que no se tiene por objetivo garantizar y mejorar la salud, educación y seguridad de las personas así como la calidad y eficiencia del servicio. Asimismo, tal y como determina Campos (2010), la falta de titulados en el mercado laboral no puede ser una justificación para no seleccionar a personas tituladas en la actividad física y deporte, ya que en la actualidad en este mercado laboral existen muchas personas tituladas en situación de paro o trabajando en sectores que no son de la actividad física y del deporte debido, entre otras causas, a que en la última década el número de titulados 
de la actividad física y del deporte graduados por año se ha multiplicado por seis. Igualmente, un mayor coste de los titulados no puede ser una argumentación para no seleccionar a titulados de la actividad física y del deporte, ya que Campos $(2003,2007)$ y González (2008) han obtenido que no se producen mayores ingresos de los titulados sobre los no titulados ni tampoco que a mayor titulación se tiene mayores ingresos. Igualmente, como explica Jiménez (200l), habrá que tener en cuenta la responsabilidad civil y penal de las personas que trabajan sin la titulación adecuada y las entidades que los selecciona. También hay que enfatizar que se incumple lo establecido en distintas Leyes del deporte de diferentes Comunidades Autónomas respecto a la exigencia de posesión de la correspondiente titulación de la actividad física y del deporte (Campos y González, 2009).

Por todo ello, es importante que el proceso de selección se realice de forma adecuada, en el que los instrumentos utilizados y procesos realizados tengan validez y fiabilidad y éstos posean la titulación de la actividad física y del deporte correspondiente, aunque además de este criterio esencial se debe valorar posteriormente otros aspectos. Asimismo, es necesaria la existencia de un adecuado desarrollo legislativo y laboral, en la que se estipule que los distintos agentes empleadores han de cumplir los mínimos requisitos de exigencia de titulación de la actividad física y del deporte y laborales, así como un cuerpo de inspectores que velen por el cumplimiento de dicha normativa. Al respecto en Cataluña en el año 2008 se ha aprobado la ley del ejercicio de las profesiones del deporte, cuyos efectos y evolución todavía no han podido ser estudiados en profundidad debido a su reciente aprobación, y en la actua- lidad el Consejo Superior de Deportes está realizando el anteproyecto de Ley sobre ordenación del ejercicio de determinadas profesiones de deporte.

\subsection{Los instrumentos utilizados en la selección respecto a la formación inicial}

En el año 2004, tanto en la selección de las personas que poseen alguna titulación de la actividad física y del deporte como en las personas que no la poseen, se ha utilizado toda la gama de instrumentos obtenidos en el estudio, tanto de forma individual como combinada (tabla 4). En las referencias o contactos, la entrevista y la utilización conjunta de entrevista y referencias se selecciona mayoritariamente a personas que no poseen ninguno de los diferentes tipos de titulaciones de la actividad física y del deporte, ya que representan el $65,3 \%, 56,7 \%$ y el $55,6 \%$ respectivamente. Sin embargo, en el currículum vitae, el currículum vitae junto con la entrevista, el currículum vitae con las referencias y el currículum vitae junto con la entrevista y las referencias se seleccionan mayoritariamente a personas que poseen alguno de los diferentes tipos de titulaciones de la actividad física y del deporte, ya que representan el 54,2\%, 66,7\%, $57,1 \%$ y $66,7 \%$ respectivamente (Tabla 7 ). El instrumento de selección que mayor diferencia existe de las personas no tituladas sobre las tituladas de la actividad física y del deporte es las referencias o contactos, al igual que obtienen Martínez del Castillo (1991) y González (2008). Por el contrario, donde mayor diferencia existe de las personas tituladas sobre las no tituladas de la actividad física y del deporte son los instrumentos de selección de currículum vitae junto con la entrevista y el currículum vitae jun-

Tabla 7

Los instrumentos utilizados en la selección respecto a la formación inicial en 2004

\begin{tabular}{|l|c|c|}
\hline & $\begin{array}{c}\text { No titulados de la actividad } \\
\text { física y del deporte (\%) }\end{array}$ & $\begin{array}{c}\text { Titulados de la actividad } \\
\text { física y deporte (\%) }\end{array}$ \\
\hline Currículum vitae & 45,8 & 54,2 \\
\hline Referencias o contactos & 65,3 & 34,7 \\
\hline Entrevista & 56,7 & 43,3 \\
\hline Currículum vitae/entrevista & 33,3 & 66,7 \\
\hline Currículum vitae/referencias o contactos & 42,9 & 57,1 \\
\hline Entrevista/ referencias o contactos & 55,6 & 44,4 \\
\hline Currículum vitae/entrevista/referencias o contactos & 33,3 & 66,7 \\
\hline
\end{tabular}


to con la entrevista y las referencias o contactos. Se obtiene una relación alta $(\mathrm{Phi}=0,788)$ y significativa (Chi-cuadrado $=146,531 ; p=0,00)$.

En el año 2006, tanto en la selección de las personas que poseen alguna titulación de la actividad física y deporte como en las personas que no la poseen, se ha utilizado toda la gama de instrumentos obtenidos en el estudio y ha sido tanto de forma individual como combinada (tabla 5). En las referencias o contactos, la entrevista y la utilización conjunta de entrevista y referencias se selecciona mayoritariamente a personas que no poseen ninguno de los diferentes tipos de titulaciones de la actividad física y del deporte ya que representan el $66,7 \%, 60 \%$ y el $57,1 \%$ respectivamente. Sin embargo, en el currículum vitae, el currículum vitae junto con la entrevista, el currículum vitae junto con las referencias y el currículum vitae junto con la entrevista y las referencias se selecciona mayoritariamente a personas que poseen alguno de los diferentes tipos de titulaciones de la actividad física y del deporte, debido a que representan el 64\%, 68,3\%, 56,2\% y 66,7\% respectivamente (Tabla 8).

El instrumento de selección que mayor diferencia existe de las personas no tituladas sobre las tituladas de la actividad física y del deporte es las referencias o contactos, al igual que obtienen Martínez del Castillo ( |99|) y González (2008), por el contrario existe una mayor diferencia de las personas tituladas sobre las no tituladas de la actividad física y del deporte en el currículum vitae junto con la entrevista. Se obtiene una relación moderada (Phi=0,508) pero no significativa (Chi-cuadrado $=59,658 ; p=0,121$ ).

\section{Conclusiones}

En este estudio se obtiene que más de la mitad de los recursos humanos de la actividad física y del deporte trabajan para empresas, e incluso se produce la tendencia de un gran aumento en las últimas décadas y del año 2004 al 2006. Además, se observa que el empleo por cuenta propia en los servicios de actividad física y deporte está en crecimiento.

El reclutamiento es totalmente realizado por las propias empresas, cuestión importante a tener en cuenta a la hora de dirigirlas y organizarlas. Además, el tipo de reclutamiento utilizado por las empresas es esencialmente externo, donde la utilización de Internet en dicho proceso es un método de expansión y crecimiento debido a la reducción de costes económicos y temporales.

Respecto a la selección, hay que remarcar que esencialmente se utilizan como instrumentos el currículum vitae, las referencias o contactos y la entrevista, lo que determina la visión tradicional en las empresas de dicho proceso, y en la que generalmente sólamente se utiliza un instrumento. Asimismo, hay que incidir en que no se utiliza la variedad de tipos de instrumentos utilizados en otras actividades empresariales y económicas, donde además es inexistente la selección orientada hacia las competencias.

En lo referente a la formación inicial, se puede observar claramente cómo la mayoría de los recursos humanos de la actividad física y del deporte no tienen la correspondiente titulación de la actividad física y del deporte, lo que determina, junto al elevado número de personas seleccionadas por referencias

Tabla 8

Los instrumentos utilizados en la selección respecto a la formación inicial en 2006

\begin{tabular}{|l|c|c|}
\hline & $\begin{array}{c}\text { No titulados de la actividad } \\
\text { física y del deporte (\%) }\end{array}$ & $\begin{array}{c}\text { Titulados de la actividad } \\
\text { física y deporte (\%) }\end{array}$ \\
\hline Currículum vitae & 36 & 64 \\
\hline Referencias o contactos & 66,7 & 33,3 \\
\hline Entrevista & 60 & 40 \\
\hline Currículum vitae/entrevista & 31,7 & 68,3 \\
\hline Currículum vitae/referencias o contactos & 43,8 & 56,2 \\
\hline Entrevista/ referencias o contactos & 57,1 & 42,9 \\
\hline Currículum vitae/entrevista/referencias o contactos & 33,3 & 66,7 \\
\hline
\end{tabular}


o contactos, que el proceso de selección de los recursos humanos de la actividad física y del deporte en las empresas en la ComunidadValenciana en muchos casos es inadecuado y con escasa fiabilidad y validez.

En toda la gama de tipos de instrumentos obtenidos en el estudio, tanto de forma individual como combinada, se ha seleccionado a personas que poseen alguna de las diferentes titulaciones de la actividad física y del deporte como a personas que no las poseen. El instrumento de selección que mayor diferencia existe de las personas no tituladas sobre las tituladas de la actividad física y del deporte es las referencias o contactos, por el contrario los que mayor diferencia existe de las personas tituladas sobre las no tituladas de la actividad física y del deporte es la utilización de currículum vitae junto con la entrevista.

Por todo ello, es importante que el proceso de selección de los recursos humanos de la actividad física y del deporte en las empresas se realice de forma adecuada, en el que los instrumentos utilizados y procesos realizados tengan validez y fiabilidad y que los recursos humanos de la actividad física y del deporte posean la titulación de actividad física y deporte correspondiente, aunque además de este criterio esencial se debe valorar posteriormente otros aspectos. Todo ello con el fin de garantizar la salud, educación y seguridad de las personas así como la calidad y eficiencia de la dirección y organización de las empresas y de los propios servicios de la actividad física y del deporte. Por ello, les necesario orientar a las empresas a realizar adecuadamente el proceso de selección.

\section{Bibliográfica}

ALVIRA, F. (2004). La encuesta: una perspectiva general metodológica. Ed. C. I. S., Madrid.

ANDREFF, W. y WEBER, W. ( 1 995). Economía, en HERNÁNDEZ, J.L. (Ed.), La función social del deporte en la sociedad. M.E.C., Madrid, pp. I53-182.

AUGUSTIN, J.P. (2003). Le sport et ses métiers. Ed. La Découverte, Paris.

BAYÓN, F. (2002). Organizaciones y recursos humanos. Ed. Síntesis, Madrid.

BECKER, B., HUSELID, M. y ULRICO, D. (200I). El cuadro de mando de recursos humanos. Ed. Gestión 2000, Barcelona.
BOYATZIS, R.E. ( 1 982). The competent manager: A model for effective performance. Ed. John Wiley \& Sons, New York.

BRYMAN, A. (2004). Social research methods. Ed. Oxford University Press, New York.

CAMPOS, A. (2000). Calidad en el mercado de la Actividad Física y el Deporte desde la perspectiva de ordenación adecuada de los recursos humanos», en FUENTES, J.P. y MACIAS, M. (coord.), I Congreso de la Asociación Española de Ciencias del Deporte. Universidad de Extremadura, Caceres, pp. 45 I-460.

CAMPOS, A. (200I). El mercado laboral de los profesionales de la actividad física y deporte y su regulación, en CARRATALÁ,V., GUZMÁN J.F.y FUSTER, M.A. (coord.), Il Congreso de la Asociación Española de Ciencias del Deporte. Universidad de Valencia,Valencia, pp. 993-I00I.

CAMPOS, A. (2003). La organización de la actividad física y del deporte en las instalaciones deportivas de la Comarca Burgalesa de la Ribera del Duero respecto a los profesionales de la actividad física y del deporte. Universidad de Valencia, Valencia.

CAMPOS, A. (2004). Los profesionales de la actividad física y deporte y la organización-regulación de su mercado laboral. Actividad física: ciencia y profesión, N. 5 , pp. |4-19.

CAMPOS, A. (2007). Situación profesional de las personas que trabajan en funciones de actividad física y deporte en la Comunidad Autónoma Valenciana (2005). Universidad de Valencia, Valencia.

CAMPOS, A. y GONZÁLEZ, M. D. (2009). The labour market of the professionals of the physical activity and of the sport in Spain, Esporte e Sociedade, N. II.

CAMPOS, A. (2010). Dirección de recursos humanos en las organizaciones de actividad física y deporte. Ed. Síntesis, Madrid.

CAMY, J.; CHANTELAT, P.y LE ROUX, N. (1999). Sport et emploi en Europe. Ed. Comisión Europeenne, France.

CAPELLERAS, J.L.; GENESCÁ, E. (2004). Un análisis comparativo de las características de las microempresas en España, Universia Business Review, N.2, pp. 72-93.

CEA, M. A. (1998). Metodología cuantitativa. Estrategias y técnicas de investigación social. Ed. Síntesis, Madrid.

CHIAVENATO, I. (2006). Introducción a la teoría general de la administración. Ed. McGraw-Hill, Madrid.

CHIAVENATO, I. (2007). Administración de recursos humanos. Ed. McGraw-Hill, Mexico.

CLAVER, E., GASCÓ, J.L. y LLOPIS, J. (1996). Los recursos humanos en la empresa: un enfoque directivo. Ed. Civitas, Madrid. 
COHEN, G. (|99|). La naturaleza de la función directiva. Ed. Díaz de Santos, Madrid.

COMISIONES OBRERAS (2000). Informe cuantitativo y cualitativo de gimnasios y piscinas. Ed. Comisiones Obreras, Madrid.

CONSEJO SUPERIOR DE DEPORTES (2000). EI deporte español ante el siglo XXI. Ed. Consejo Superior de Deportes-Ministerio de Educación y Cultura, Madrid.

CONSEJO SUPERIOR DE DEPORTES (2009). Borrador de anteproyecto de Ley sobre Ordenación del Ejercicio de determinadas Profesiones del Deporte. Ed. Consejo Superior de Deportes, Madrid.

CORBETTA, P. (2003). Metodología y técnicas de investigación social. Ed. McGraw Hill, Madrid.

DE SAÁ, P.y GARCÍA, J.M. (200I). El sistema de recursos humanos y el desarrollo de capacidades organizativas: una aplicación empírica en el sector de las cajas de ahorros españolas. Cuadernos de Economía y Dirección de la empresa, N. 8, pp. I09- 132.

DELERY, J.E. y DOTY, D.H. (1996). Modes of theorizing in strategic human resource management: tests of universalistic, contingency and configurational performance predictions, Academy of Mangement Journal, Vol. 39, N. 4, pp. 802-835.

DOLAN, S.L., VALLE, R., JACKSON, S.E. y SCHULER, R. E. (2003). La gestión de los recursos humanos. Preparando profesionales para el siglo XXI. Ed. McGraw-Hill, Madrid.

ELVIRA, M. y DÁVILA, A. (2004). Cultura y administración de recursos humanos en América Latina, Universia Business Review, N. 5, pp. 28-45.

ESPADA, M., CAMPOS, A. y GONZÁLEZ, M.D. (2009). Familia y actividades físico-deportivas extraescolares, Revista Padres y Maestros, N. 323, pp. 5-7.

FERNÁNDEZ-RÍOS, M. (1995). Análisis y descripción de puestos de trabajo: teoría, métodos y ejercicios. Ed. Díaz de Santos, Madrid.

FERNÁNDEZ-RÍOS, M. (1999). Diccionario de recursos humanos. Ed. Díaz de Santos, Madrid.

FINK, A. (1995). How to sample in surveys. Ed. Sage, Thousand Oaks.

FUNDACIÓN EUROPEA PARA LA GESTIÓN DE LA CALIDAD (2003). EFQM. Introducción a la Excelencia. Ed. EFQM, Bruselas.

GAMBAU,V. (2007). Estudio de la organización de los clubes deportivos en Galicia. Universidad de la Coruña, La Coruña.

GARCÍA FERRANDO, M. (2002). La encuesta, en ALVIRA, F., GARCÍA FERRANDO, M. e IBÁÑEZ, J. (comps.), El análisis de la realidad social. Métodos y técnicas de in- vestigación (ed. 3. ${ }^{a}$ ). Alianza editorial, Madrid, pp. |4|170.

GARRIGÓS, F. (2002). La regulación del ejercicio de la profesión como activo en el desarrollo de la economía del deporte, Actividad física: ciencia y profesión, N.2, pp. 5-8.

GASALLA, J.M. (1993). La nueva dirección de personas. Ed. Pirámide, Madrid.

GIBSON, J.L., IVANICEVICH, J.M.y DONNELLY, J.H. ( 1994). Las organizaciones. Ed. Addison-wesley, Buenos Aires.

GÓMEZ, A.M. Y MESTRE, J.A. (2005). La importancia del gestor deportivo en el municipio. Ed. INDE, Barcelona.

GONZÁLEZ, M. (2008). «El docente del deporte escolar, su formación inicial y la influencia en su intervención didáctica», en AYUNTAMIENTO DE DOS HERMANAS. (Ed.), V Congreso Nacional y III Congreso Iberoamericano de Deporte en Edad Escolar,Ayuntamiento de Dos Hermanas, Dos Hermanas (Sevilla), pp. 291-304.

HARDMAN, K. y MARSHALL, J. (2000). «The state and status of Physical Education in schools in international context», European Physical Education Review, vol. 6, N. 3, pp. 203-229.

HEINEMANN, K. (1998). «Introducción a la economía del deporte». Ed. Paidotribo, Barcelona.

INSTITUTO NACIONAL DE ESTADÍSTICA (2008). «Encuesta de Población activa». Ed. Instituto Nacional de Estadística, Madrid.

JIMÉNEZ, I. (200I). «El ejercicio profesional de las titulaciones del deporte». Ed. Bosch, Barcelona.

KOONTZ, H. y WEIHRICH, H. (2007). «Administración: una perspectiva global». Ed. McGraw-Hill, Maxico.

LEVY-LEBOYER, C. ( 1 997). «Gestión por competencias». Ed. Gestión 2000, Barcelona.

LÓPEZ,A. y LUNA-AROCAS, R. (2000). «Perfil del gestor público del deporte en la comunidad valenciana: Un análisis preliminar», Apunts. Educació Física i Esports , N.6I, pp. 88-94.

LUNA-AROCAS, R. (2000). «Bases para la dirección de entidades deportivas». Ed. Promolibro, Valencia.

LUSSIER, R.N. y KIMBALL, D.C. (2008). «Applied sport management skills». Ed. Human Kinetics, Champaign.

MACLEAN, J. (200I). «Perfomance appraisal for sport and recreation managers». Ed. Human Kinetics, Champaign.

MADELLA, A. (2002). «Le professioni dello sport». SDS: Rivista di Cultura Sportiva, N. 55, pp. 2-9.

MADELLA, A. (2003). «Methods for analysing sports employment in Europe», Managing Leisure, Vol. 8, N. 2, pp. 56-69. 
MARTÍNEZ DEL CASTILLO, J. ( 199 I). «La estructura ocupacional del deporte en España. Encuesta realizada sobre los sectores de Entrenamiento, Docencia, Animación y Dirección». Ed. Consejo Superior de Deportes, Madrid.

MARTÍNEZ DEL CASTILLO, J. ( 1992). «Proyección del mercado deportivo laboral en la España de los noventa. En los sectores de entrenamiento, docencia, animación y dirección». Ed. Consejo Superior de Deportes, Madrid.

MARTÍNEZ DEL CASTILLO,J. ( 1997). «El proceso de profesionalización de los recursos humanos de las asociaciones deportivas de España», Apunts. Educació Física i Esports, N.49, pp. 63-83.

MARTÍNEZ, G., CAMPOS, A., PABLOS, C. y MESTRE, J.A. (2008). «Los recursos humanos de la actividad física y del deporte. Funciones y características socio-demográficas, laborales y formativas». Ed.Tirant lo Blanch, Valencia.

MCCLELLAND, D.C. ( 1973). «Testing for competence rather than for intelligence», American Psychologist, Vol. 28, N. I, pp. I - 14.

MCMURTIE, R. (1997). «Sports employment trends in Australia», Sport Educator, Vol. 9, N.2, pp. 23-24.

MIQUEL, S., BIGNÉ, E., CUENCA, A.C., LÉVY, J.y MIQUEL, M.J. (2000). «Investigación de Mercados». Ed. McGrawHill, Madrid.

MORENO, J.A. y GUTIÉRREZ, M. ( 1999). «Perfil de los educadores de programas acuáticos». Revista Española de Educación Física y Deporte, Vol. 6, N. I, pp. 12-23.

PAZ, M., (2004). «Nuevos procedimientos en el proceso empresarial de provisión de candidatos: el reclutamiento «on line»», Cuadernos de Ciencias económicas y empresariales, N. 47, pp. 89-1 10

PEIRÓ, J.M. ( I 992). «Psicología de las organizaciones. Tomo |». Ed. UNED, Madrid.

PEIRO, J. M., MARTÍNEZ-TUR, V. y TORDERA, N. (1999). «Análisis del puesto de gerente de instalaciones deportivas: tareas reactivas y preactivas». Anuario de psicología,Vol. 30, N. I, pp. 85- 03.

PEREDA, S. y BEROCAL, F. (200 I). «Técnicas de gestión de recursos humanos por competencias». Ed. Centro de estudios Ramón Areces, Madrid.

PEREDA, S. y BERROCAL, F. (1999): Gestión de recursos humanos por competencias. Ed. Centro de estudios Ramón Areces, Madrid.

PEREZ, M.P y GARRIDO, M.J. (2006). «La investigación en recursos humanos en España», Investigaciones europeas de dirección y economía de la empresa,Vol. 12, N. 2, pp. | 19-136.
PFEFFER, J. (1994). «Competitive advantage through people», California Management Review,Vol. 36, N. 2, pp. 929.

PIN, J.R.; LAORDEN, M. y SÁENZ-DÍEZ, I. (2002). «Reclutamiento a través de Internet: Oportunidades y riesgos», Harvard Deusto Business Review, N. I06, pp. 64-69.

PIRES, G. (2007). «Agôn. Gestao do desporto». Ed. Porto. Porto.

PORRET, M. (2006). «Recursos humanos. Dirección y gestión de personas en las organizaciones». Ed. ESIC, Madrid.

PUIG, N. (1980). «Proposiciones metodológicas para el análisis de la formación del profesorado de Educación Física y de su «status» profesional en España de 1939 a la actualidad»), Apunts de medicina del deporte, N. 66, pp. 85-90.

PUIG, N. y VIÑAS, J. (200I). « Mercat de treball i llicenciatura en Educació Física a I'INEF-Catalunya, Barcelona ( 1980- 1997)». Ed. INEFC-Cataluña, Barcelona.

PUIG, N. y VIÑAS, J. (2006). «Estructura ocupacional de Licenciados y Licenciadas en el INEF-Cataluña, Barcelona (1980-2005)». Ed. INEFC-Cataluña, Barcelona.

RAMOS J. (1993). «Modelos de gestión de instalaciones deportivas en la Comunidad Valenciana», Universidad de Valencia, Valencia.

RENAU, J.J. ( I 985). «Administración de empresas. Una visión actual». Ed. Pirámide, Madrid.

RIVADENEYRA, M.L. (2000). «Análisis de la especialización de los Licenciados de la Actividad Física y Deporte en relación con las demandas del mercado laboral deportivo», en FUENTES J.P.y MACIAS, M. (Coords.), I Congreso de la asociación Española de Ciencias del deporte, Tomo II, Universidad de Extremadura, Cáceres, pp. 5 | 3-520.

RODRÍGUEZ, J. (|99|). «Métodos de muestreo». C.I.S. Madrid.

SALGADO, J.F. y MOSCOSO, S. (2008). «Selección de personal en la empresa y la AAPP: de la visión tradicional a la visión estratégica», Papeles del psicólogo, Vol. 29, N. I, pp. 16-24.

SALGADO, J.F.; MOSCOSO, S. y LADO, M. (2004). «Reclutamiento y selección», en BONACHE, J.Y CABRE$R A, A$. (dir.), Dirección estratégica de personas, Parsons Educación, Madrid, pp. 91-134

SASTRE, M.A. y AGUILAR, E.M. (2003). «Dirección de recursos humanos: un enfoque estratégico». Ed. McGrawHill, Madrid.

SEIRUL-LO, F. ( 1 987). «Las funciones y competencias del preparador físico en un club deportivo», Revista de Entrenamiento Deportivo, Vol. I, N. I, pp. 70-77. 
SHIBLI, S.;TAYLOR, P. NICHOLS, G. GRATTON, C. y KOKOLAKAKIS,T. (1999). «The characteristics of volunteers in UK sport clubs», European Journal of Sports Management, Special Issue, pp. 10-27.

SHULER, R. (1992). «Strategic Human Resource Management:Linking the people with the strategic needs of Business», Organizational Dynamics, Vol. 21, N. I, pp. 1832.
SIERRA, R. (200I). «Técnicas de investigación social.Teoría y ejercicios». Ed. Paraninfo, Madrid.

SOUCIE, D. (2002). «Administración, organización y gestión deportiva». Ed. INDE, Barcelona.

TOJAL, J. (2004). «Da educaçao Física à Motricidade Humana. Preparaçao do profisional». Ed. Instituto Piaget, Lisboa. 